\title{
An ODF-Analysis Program for Orthorhombic Crystal Symmetry and its Application to $\mathrm{CuZnAl}$ Shape-Memory-Alloys
}

No Jin Park and Hans Joachim Bunge Department of Physical Metallurgy, TU Clausthal Großer Bruch 23, D-3392 Clausthal-Zellerfeld, FRG

\section{Introduction}

In the last few years texture analysis was extended from basic metals, mainly with cubic and hexagonal structure, to materials such as intermetallic phases e.g. shape memory alloys, ceramics, e.g. HTcsuperconductors or polymers. These materials often have lower crystal symmetries and more complex diffraction spectra with partially or totally overlapping diffraction peaks. Therefor, new methods of ODF calculation had to be developed taking these conditions into account. In the present work an ODF program for orthorhombic crystal and sample symmetry was developed which accepts incomplete and superposed pole figures. The program is based on the series expansion method. The positivity condition is taken into account by an iteration process. The program was applied to study texture development in a shape memory alloy in the CuZnAl system. In CuZnAl-alloys shape memory effects are observed in the vicinity of $14 \% \mathrm{Zn}$ and $6 \% \mathrm{Al}$. The high temperature phase is b.c.c whereas the low-temperature phase is monoclinic. The monoclinic angle $\beta$ deviates, however, only very little, i.e. $1.21^{\circ}$ from $90^{\circ}$. Hence, for a first investigation the symmetry can be considered to be orthorhombic. 


\section{Mathematical fundamentals}

The orientation distribution function $f(g)$ of the crystallites of a polycrystalline material is defined by the volume fraction $d V / V$ of crystals which have an orientation $g$ within the range $d g$

$$
f(g)=\frac{d V(g) / V}{d g} \quad ; \quad g=\left\{\varphi_{1}, \Phi, \varphi_{2}\right\}
$$

The function $f(g)$ can be obtained from pole figure measurement followed by pole figure inversion (ODF-analysis)

$$
P_{\mathbf{h}}(\mathbf{y})=\frac{1}{2 \pi} \int_{\mathbf{h} \| \mathbf{y}} f(g) d \psi
$$

where $\mathbf{h}$ is the crystal direction and $\mathbf{y}$ is the sample direction.

To solve eq. 2 for $f(g)$ with several given pole figures, $f(g)$ is expressed in terms of a series expansion

$$
f(g)=\sum_{l=0}^{l_{\max }} \sum_{\mu=1}^{M(l)} \sum_{\nu=1}^{N(l)} C_{l}^{\mu \nu} \cdot \ddot{T}_{l}^{\mu \nu}(g)
$$

This leeds eq.2 to the following form

$$
P_{\mathbf{h}}(\mathbf{y})=\sum_{l=0}^{l \max } \sum_{\mu=1}^{M(l)} \sum_{\nu=1}^{N(l)} \frac{4 \pi}{2 l+1} C_{l}^{\mu \nu} \cdot \dot{k}_{l}^{* \mu}(\mathbf{h}) \cdot \dot{k}_{l}^{\nu}(\mathbf{y})=R_{\mathbf{y}}(\mathbf{h})
$$

The pole figure $P_{\mathbf{h}}(\mathbf{y})$ is the distribution of a particular crystal direction $\mathbf{h}$ with respect to the sample coordinate system.

If, on the other hand, one keeps a particular sample direction y constant and takes the crystal direction $h$ as variable then eq. 4 describes the inverse pole figure, which indicates the frequencies with which different crystal directions occur in a specified sample direction.

The texture coefficients $C_{l}^{\mu \nu}$ can be determined from experimental pole figures by solving eq. 4 .

Due to the experimental conditions, however, the following complications must also be taken into account:

- The pole figures are being measured incompletely. In order to deal 
with this difficulty it is useful to take the necessary positivity condition $P_{\mathbf{h}}(\mathbf{y}) \geq 0$ into account which must be fulfilled for all $(h k l)$ including those which were not measured as pole figures.

- Pole figures with different $(h k l)$ may be superposed at the same Bragg-angle $\theta$. Hence, the measured pole figures

$$
P_{\theta}(\mathbf{y})=\sum_{i=1}^{I} q_{i} \cdot P_{\mathrm{h}_{i}}(\mathbf{y})
$$

are superpositions of the ones defined by eq.4. The superposition factors $q_{i}$ are calculated by an iteration process in the pole figure inversion procedure.

- The coefficients $C_{l}^{\mu \nu}$ with odd l-values cannot be obtained from eq.4. The odd coefficients can, however, be obtained by using the necessary positivity of the function $f(g) \geq 0$ in a second iterative process.

This procedure is illustrated schematically in Fig.1.

\section{Experimental Results}

The alloy $\mathrm{Cu}-14.2 \mathrm{wt} \% \mathrm{Zn}-5.6 \mathrm{wt} \% \mathrm{Al}$ was melted in a medium frequency induction furnace and hot rolled at $750^{\circ} \mathrm{C}$ in steps of $20 \%$ to a final thickness of $1 \mathrm{~mm}$ equivalent to $96 \%$ total rolling reduction. Thereby several intermeditate annealing treatments at $750^{\circ} \mathrm{C}$ were necessary. After that the material was cooled down to room temperature whereby it transforms from b.c.c. to monoclinic.

The transformation temperatures were found to be

$$
M_{s}=115^{\circ} \mathrm{C}, \quad M_{f}=99^{\circ} \mathrm{C}, \quad A_{s}=119^{\circ} \mathrm{C}, \quad A_{f}=128^{\circ} \mathrm{C} .
$$

The lattice parameters of the monoclinic phase are

$$
\mathrm{a}=4.457 \AA, \mathrm{b}=5.326 \AA, \mathrm{c}=38.184 \AA, \beta=88.79^{\circ}
$$

Samples for texture measurement were prepared from the middle of the sheet. Twelve incomplete pole figures were measured in steps of $\Delta \alpha=5^{\circ}, \Delta \beta=3.6^{\circ}$ up to $\alpha_{\max }=70^{\circ}$ using $C o K_{\alpha}$-radiation. Six of 
the twelve pole figures were superposed ones.

The measured pole figures (after normalization) are shown in Fig.2. The ODF was then calculated with $l_{\max }=22$. The mean absolute values of the coeffients $C_{l}^{\mu \nu}$ are given in Fig.3. They are compared with their probable errors which can be estimated along with the least-squares solution. The pole figures recalculated from the obtained ODF-coefficients are shown in Fig.4. One sees that they agree quite well with the experimental ones.

The complete ODF is shown in Fig.5. With a maximum value of 31.11 it represents a rather strong texture. The texture contains several orientation components, which are given in Table 1 along with their respective densities and approximate indices of rolling plane and rolling direction.

According to the orientation relationships between b.c.c. austenit and monoclinic martensit $[100]_{M}\left\|\langle 110\rangle_{A},[010]_{M}\right\|\langle 100\rangle_{A}$, $[001]_{M} \|\langle 504\rangle_{A}$, the major components are assumed to orginate from an orientation $\{001\}\langle 011\rangle$ of the high temperature b.c.c. phase as the strongest component of the b.c.c.-rolling textur. Finally, the inverse pole figures for the rolling (RD) and normal (ND) directions are shown in Fig.6. In these figures also the orientation components A - F (Tab.1) are indicated.

\section{References}

1. K. Otsuka, K. Shimizu

Int. Metals Rev., 31, (1986), 93-114

2. H.J. Bunge

Texture Analysis in Material Science, 1982, Butterworth, London

3. M. Dahms, H.J. Bunge

J. Appl. Cryst., 22, (1989), 439-447

4. N.J.Park, H.J. Bunge

Z. Metallkunde, $\underline{81},(1990), 636-645$

5. K. Adachi, J. Perkins, C.M. Wayman

Acta Met., $\underline{36}$, (1988), 1343-1364

6. T. Saburi, S.Nenno

Proc.Int.Conf."Solid-solid phase transformation",1981, 1455-1479 


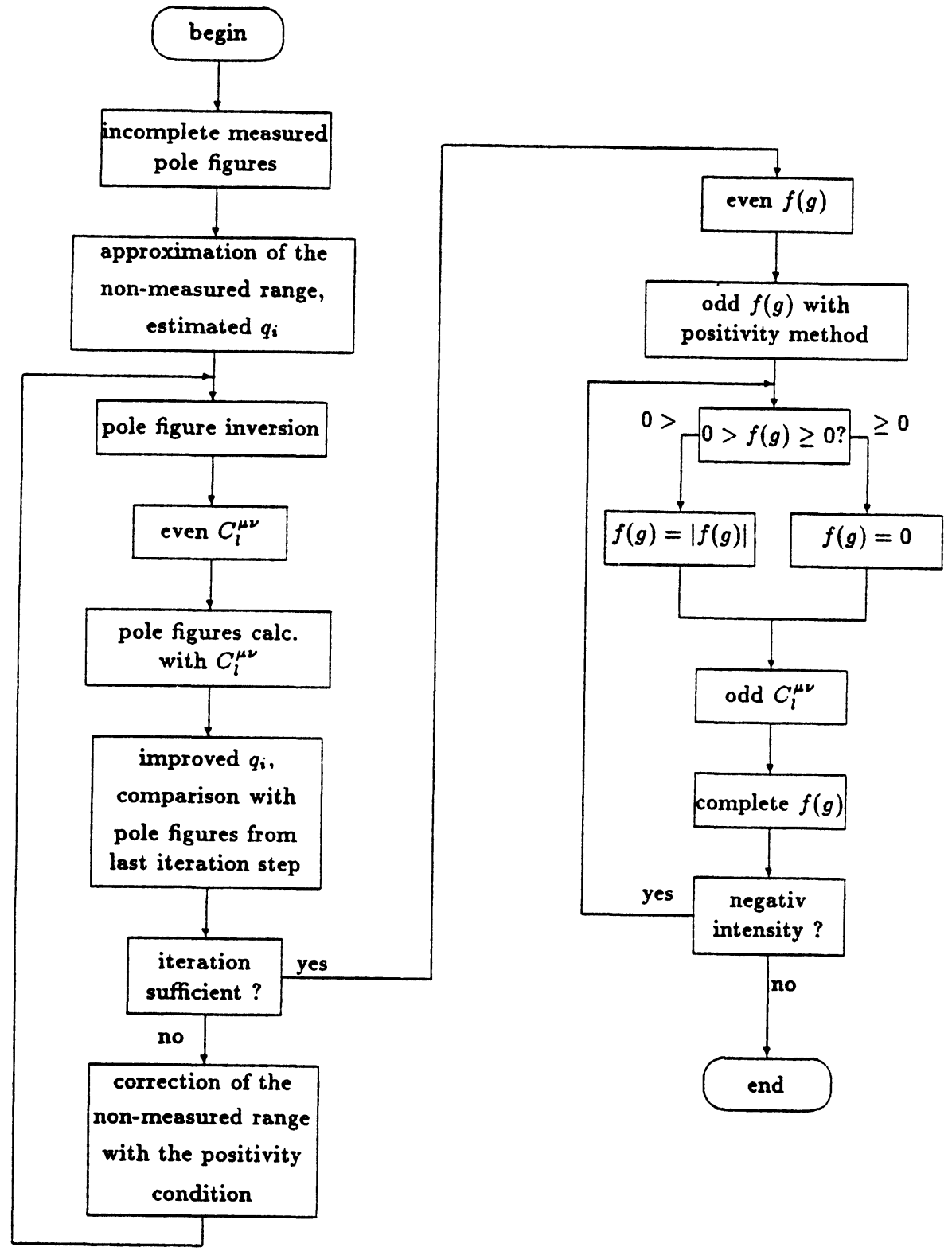

Fig.1 : Flow diagram for the calculation of the complete ODF from incomplete superposed pole figures 


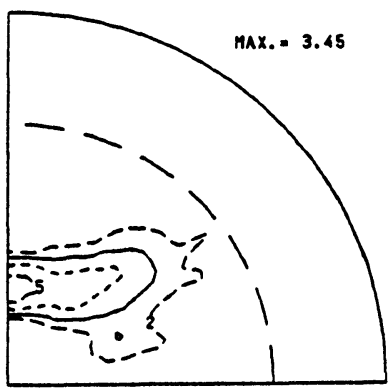

$(12 \overline{2})+(124)$

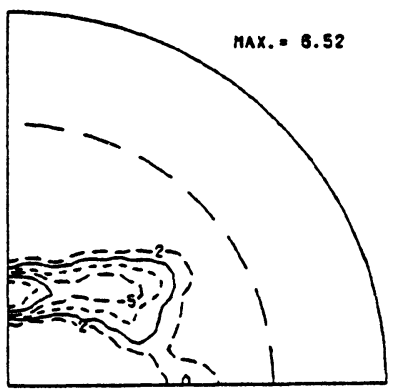

(12 10$)$

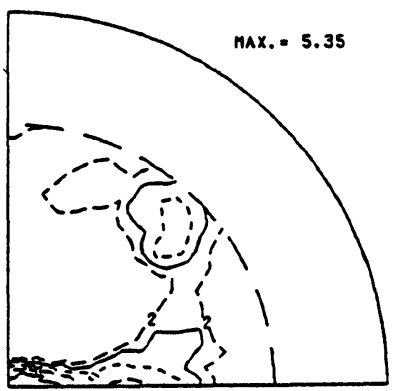

$(1220)+(2020)$

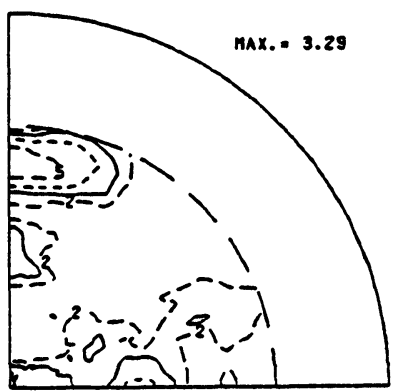

$(12 \overline{26})+(2026)$

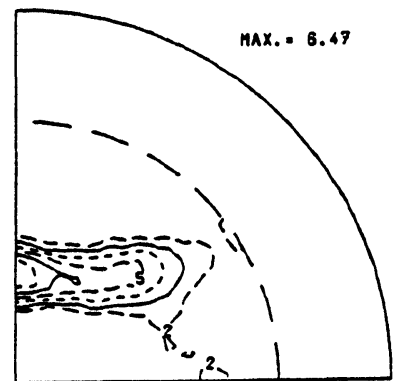

$(0018)+(20 \overline{4})$

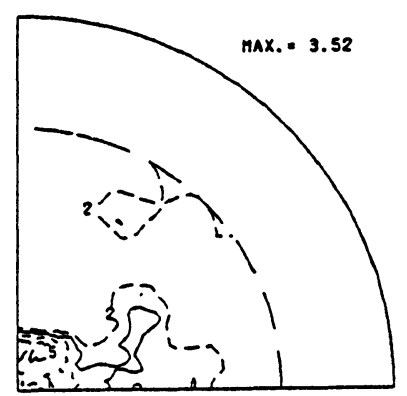

(2010)

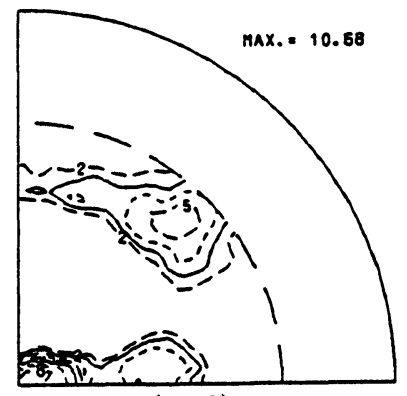

(040)

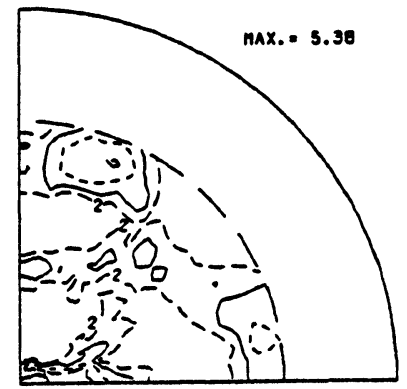

(1228)

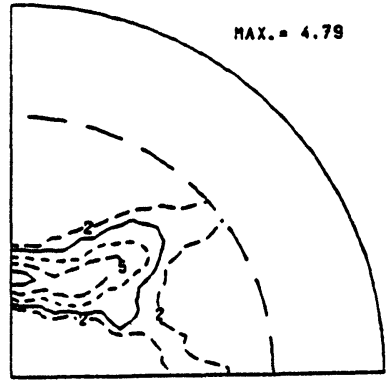

$(12 \overline{8})+(208)$

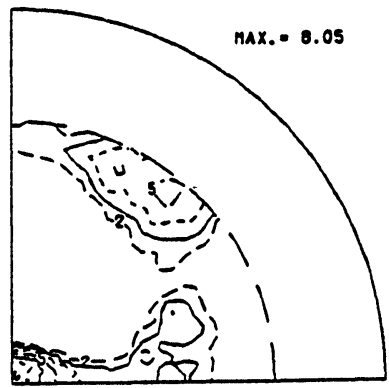

(20 $\overline{16})$

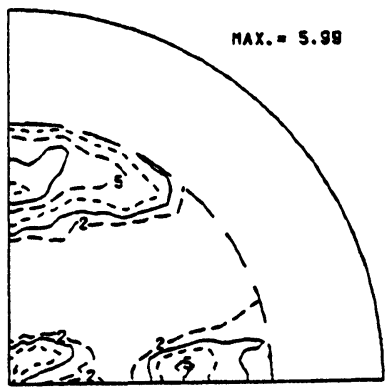

(320)

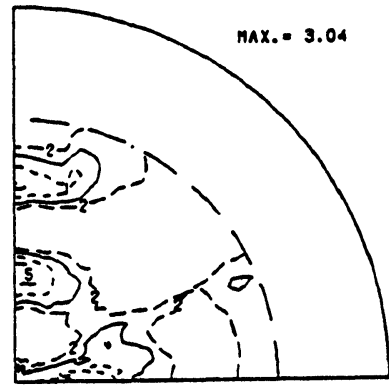

$(0418)+(24 \overline{4})$

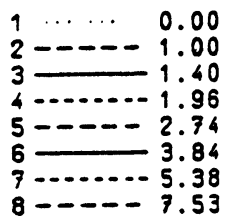

Fig.2 : Measured pole figures of an alloy $\mathrm{Cu}-14.2 \% \mathrm{Zn}-5.6 \% \mathrm{Al}$ after normalization 


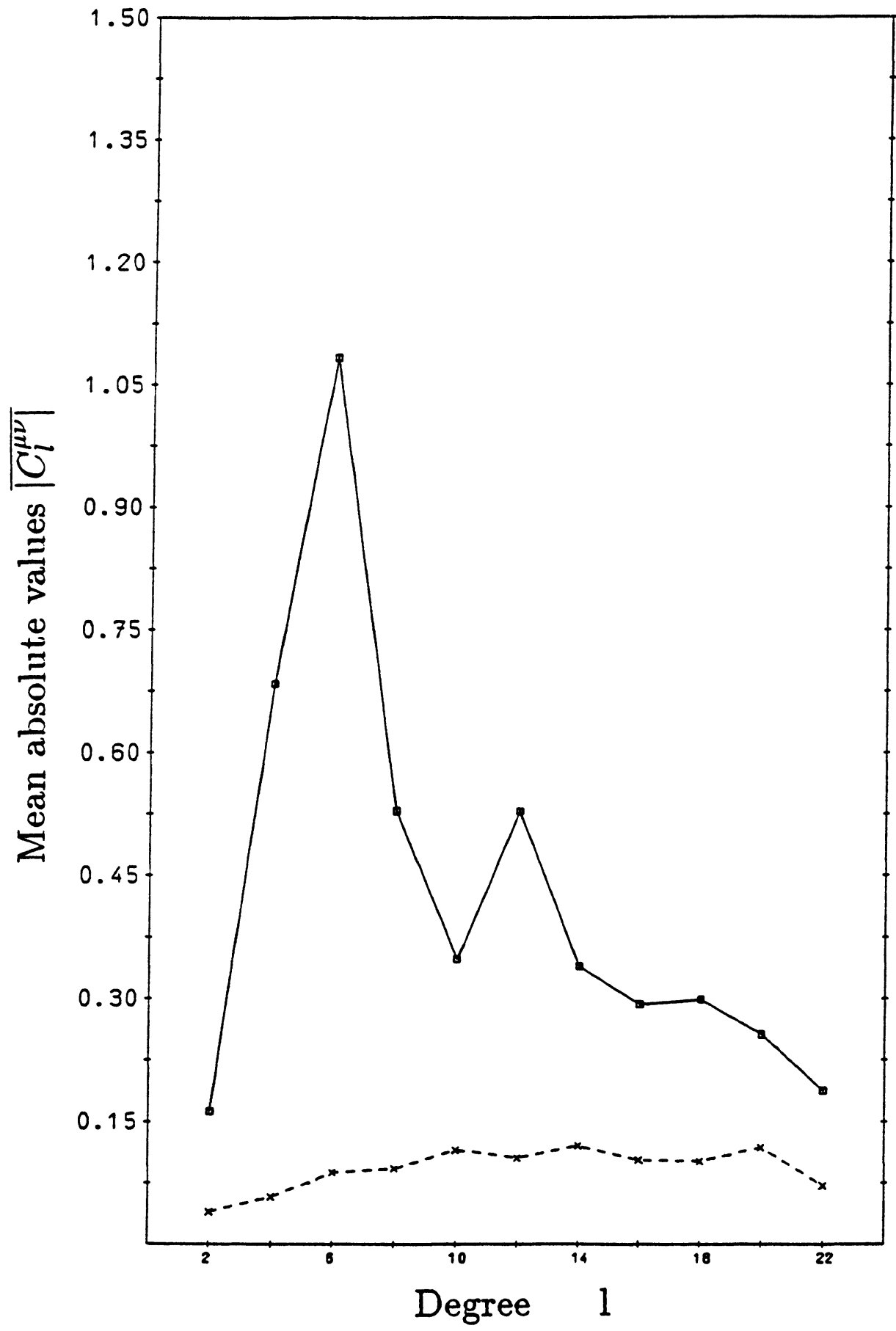

Fig.3 : Mean absolute values $\overline{\left|C_{l}^{\mu \nu}\right|}$ and their respective errors 


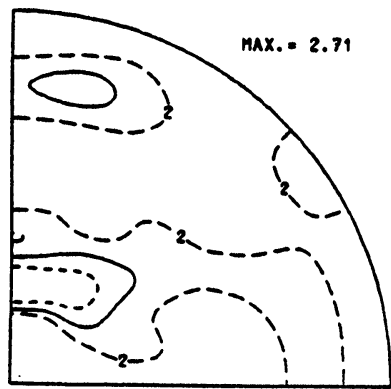

$(12 \overline{2})+(124)$
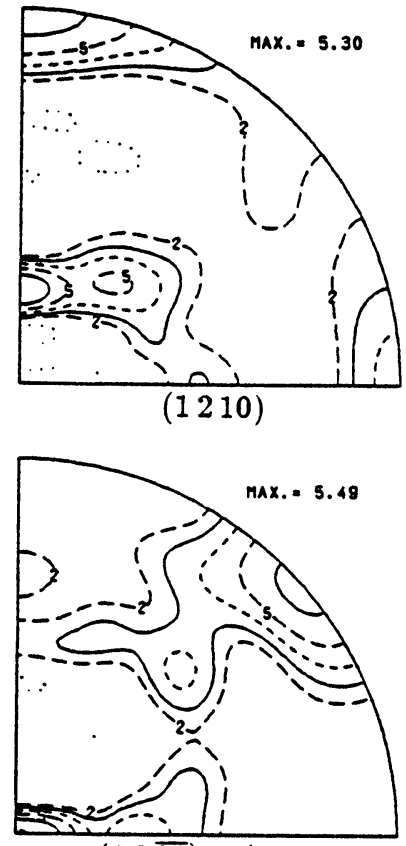

$(12 \overline{20})+(2020)$

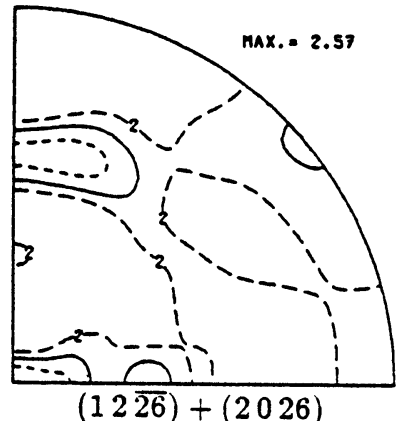

$(12 \overline{26})+(2026)$
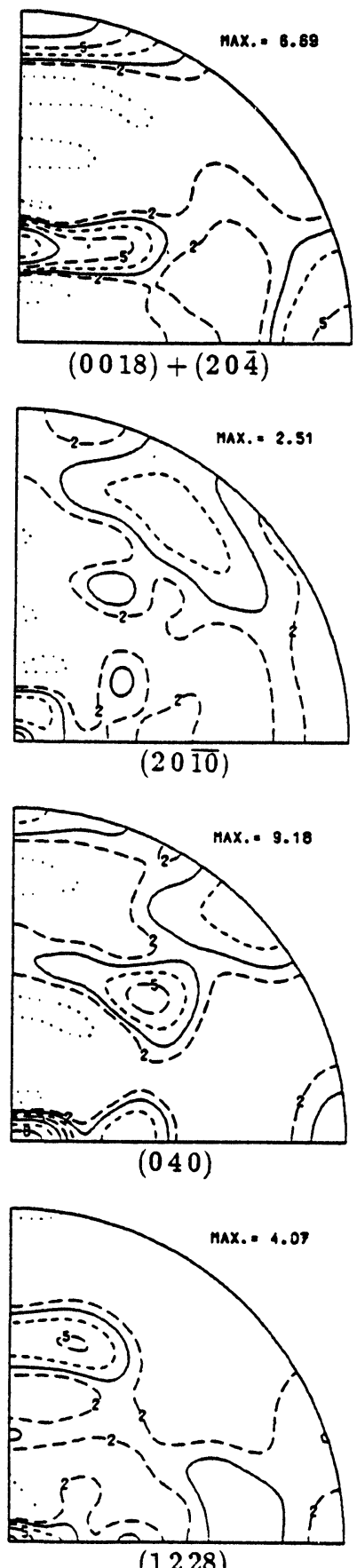

(1228)
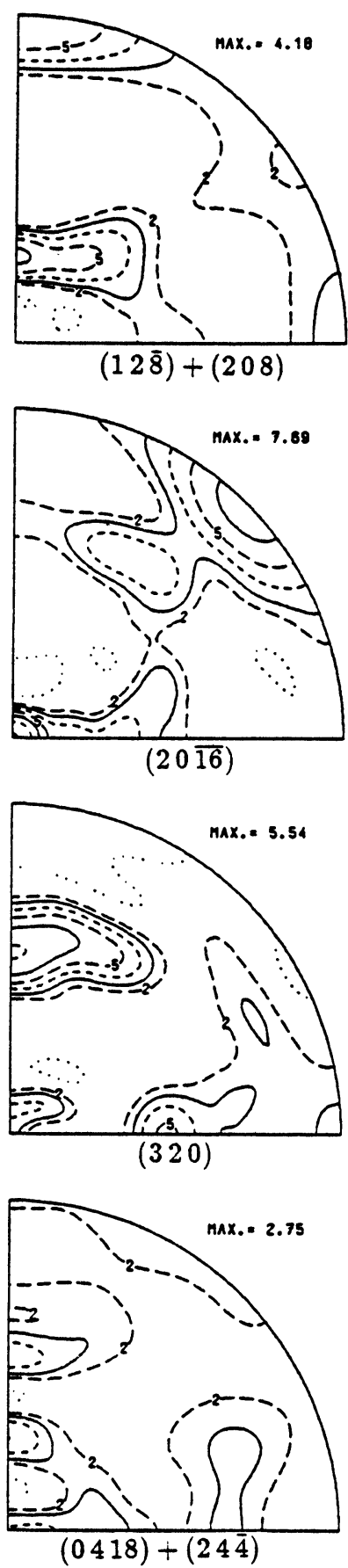

1 ......... 0.00

$2----1.00$

$3-1.40$

$4 \ldots \ldots . . .91 .96$

$5-\ldots-2.74$

$6-3.84$

$7 \ldots \ldots \ldots 5.38$

A $\ldots \ldots$. 7.53 


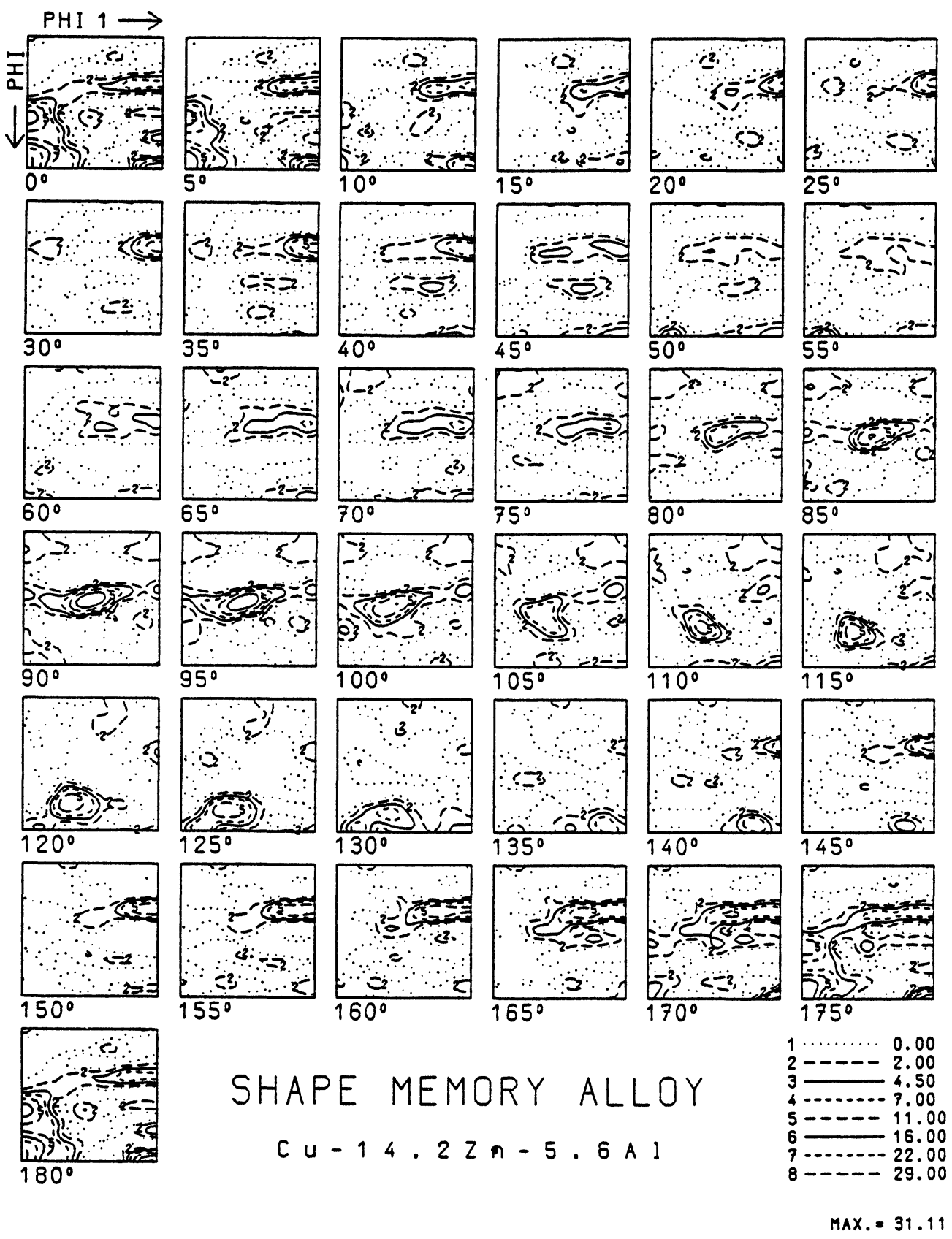

Fig.5 : ODF of hot rolled $\mathrm{Cu}-14.2 \% \mathrm{Zn}-5.6 \% \mathrm{Al}$ 

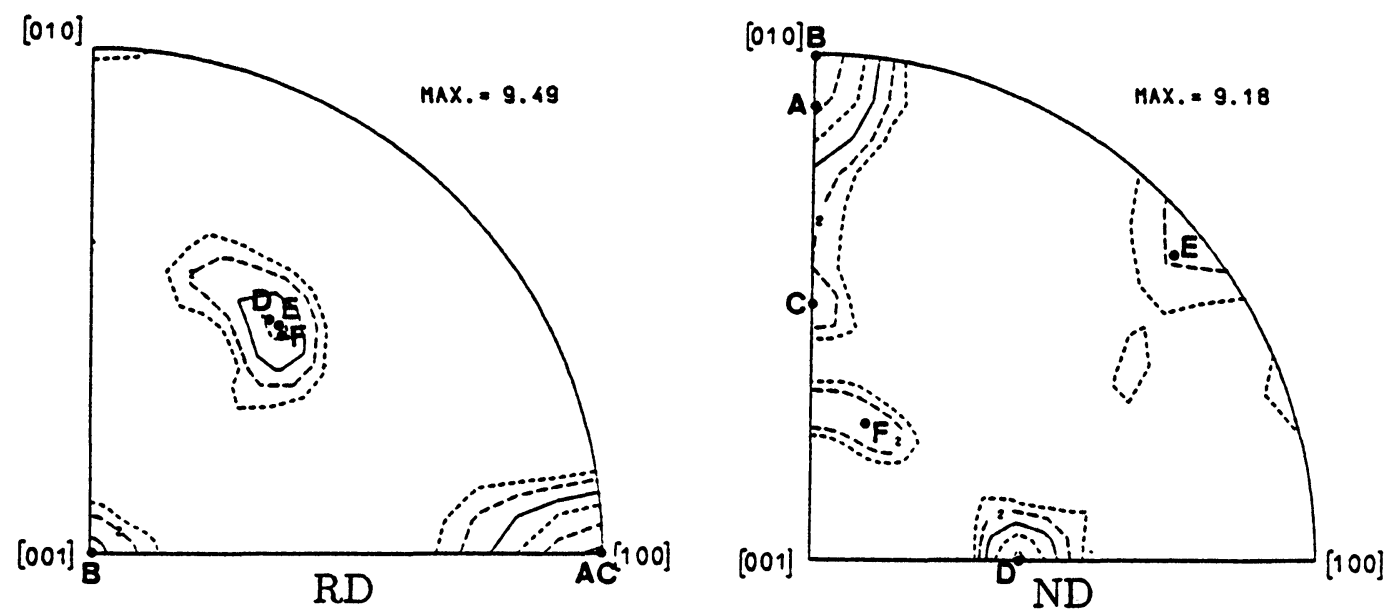

Fig.6 : Inverse pole figur of an alloy $\mathrm{Cu}-14.2 \% \mathrm{Zn}-5.6 \% \mathrm{Al}$

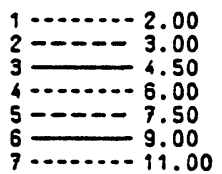

Table 1: orientation components of $96 \%$ hot rolled $\mathrm{CuZnAl}$-alloy

\begin{tabular}{|cccccc|}
\hline component & $\varphi_{1}$ & $\Phi$ & $\varphi_{2}$ & $(h k l)[u v w]$ & $f(g)$ \\
\hline A & $0^{\circ}$ & $82^{\circ}$ & $0^{\circ}$ & $(011)[100]$ & 31.1 \\
B & $90^{\circ}$ & $90^{\circ}$ & $0^{\circ}$ & $(010)[001]$ & 23.1 \\
C & $0^{\circ}$ & $54^{\circ}$ & $0^{\circ}$ & $(015)[100]$ & 18.8 \\
D & $45^{\circ}$ & $45^{\circ}$ & $90^{\circ}$ & $(109)[9.10 . \overline{1}]$ & 21.6 \\
E & $30^{\circ}$ & $73^{\circ}$ & $120^{\circ}$ & $(10 . \overline{7} .30)[10.10 . \overline{1}]$ & 14.9 \\
F & $68^{\circ}$ & $32^{\circ}$ & $160^{\circ}$ & $(1 . \overline{3} .37)[10 . \overline{9} . \overline{1}]$ & 15.2 \\
\hline
\end{tabular}

